\title{
HEALTHY AGING
}




\title{
ANNALS OF TRADITIONAL CHINESE MEDICINE
}

\author{
Series Editors: Ping-Chung Leung \\ (The Chinese University of Hong Kong, Hong Kong) \\ Charlie Changli Xue \\ (RMIT University, Australia)
}

\section{Published}

Volume 1 Chinese Medicine - Modern Practice

Edited by Ping-Chung Leung \& Charlie Changli Xue

ISBN: 978-981-256-018-6

Publication date: February 2005252 pp.

Volume 2 Current Review of Chinese Medicine: Quality Control of Herbs and Herbal Material

Edited by Ping-Chung Leung, Harry Fong \& Charlie Changli Xue ISBN: 978-981-256-707-9

Publication date: May $2006 \quad 308$ pp.

Volume 3 Alternative Treatment for Cancer

Edited by Ping-Chung Leung \& Harry Fong

ISBN: 978-981-270-929-5

Publication date: November 2007384 pp.

Forthcoming

Volume 5 Evidence-Based Acupuncture

Edited by Ping-Chung Leung

ISBN: 978-981-4324-17-5

Scheduled publication date: Fall 2011 Approx. 200 pp. 
Annals of Traditional Chinese Medicine-Vol. 4

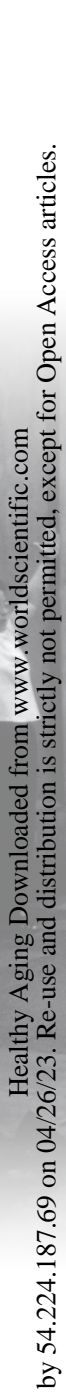
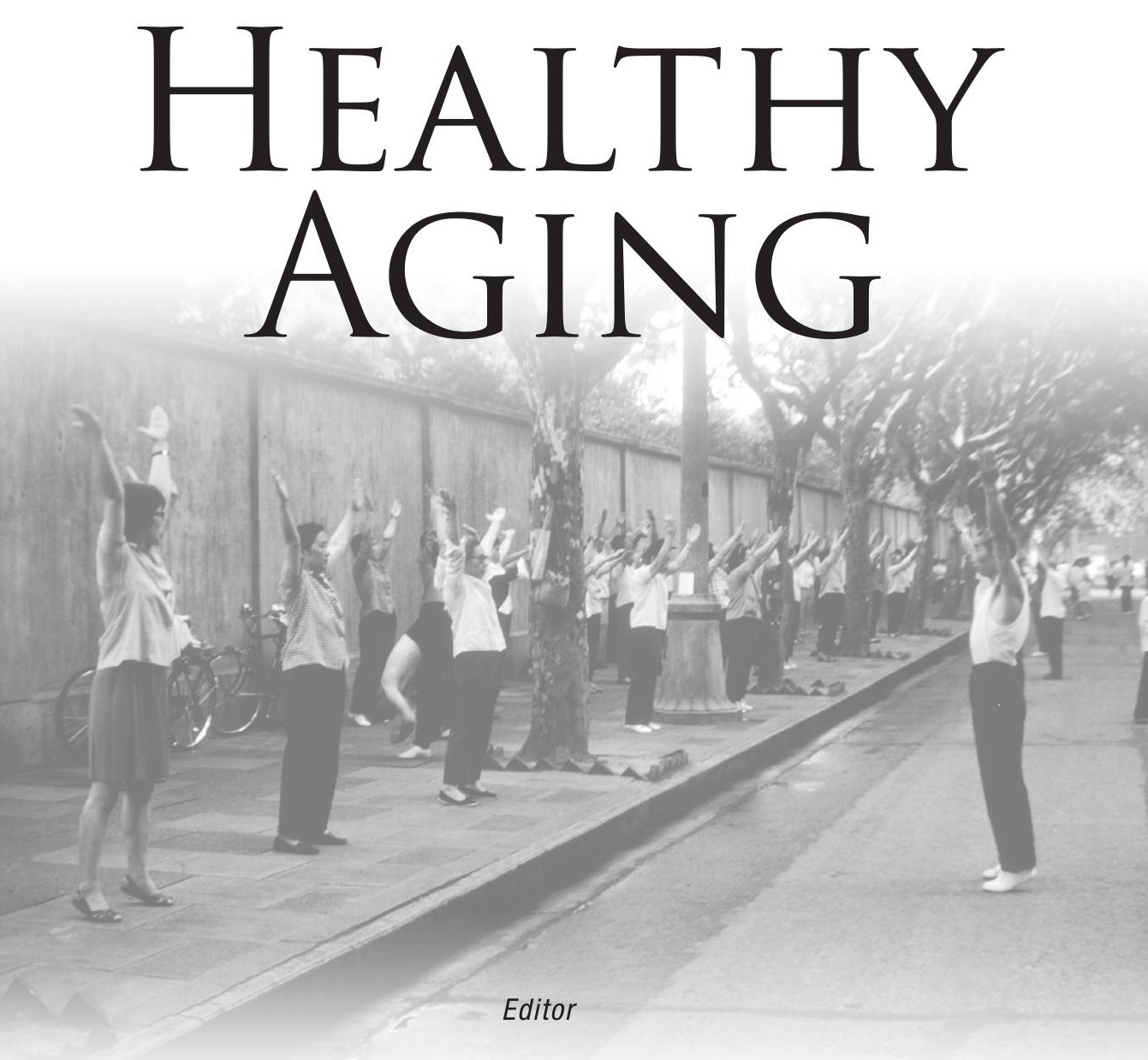

Ping-Chung Leung

The Chinese University of Hong Kong 
Published by

World Scientific Publishing Co. Pte. Ltd.

5 Toh Tuck Link, Singapore 596224

USA office: 27 Warren Street, Suite 401-402, Hackensack, NJ 07601

UK office: 57 Shelton Street, Covent Garden, London WC2H 9HE

\section{British Library Cataloguing-in-Publication Data}

A catalogue record for this book is available from the British Library.

\section{HEALTHY AGING}

Annals of Traditional Chinese Medicine - Vol. 4

Copyright () 2011 by World Scientific Publishing Co. Pte. Ltd.

All rights reserved. This book, or parts thereof, may not be reproduced in any form or by any means, electronic or mechanical, including photocopying, recording or any information storage and retrieval system now known or to be invented, without written permission from the Publisher.

For photocopying of material in this volume, please pay a copying fee through the Copyright Clearance Center, Inc., 222 Rosewood Drive, Danvers, MA 01923, USA. In this case permission to photocopy is not required from the publisher.

ISBN-13 978-981-4317-71-9

ISBN-10 981-4317-71-3

Typeset by Stallion Press

Email: enquiries@stallionpress.com

Printed in Singapore. 
Editorial Board of the Annals of Traditional Chinese Medicine

\author{
Special Advisors: \\ Ke-Ji Chen (China) \\ Seung-Hoon Choi (Philippines) \\ David Eisenberg (USA) \\ Shi-Long Lai (Hong Kong) \\ Shichen Zhang (Hong Kong) \\ Xiaorui Zhang (Switzerland) \\ Chief Editors: \\ Ping-Chung Leung (Hong Kong) \\ Harry H.-S. Fong (USA) \\ Charlie Changli Xue (Australia) \\ Executive Editors: \\ William King-Fai Cheng (Hong Kong) \\ Sim-Kim Cheng (Singapore) \\ Chye-Tee Goh (Singapore)
}

\title{
Associate Editors:
}

Alan Bensoussan (Australia)

Ji-Sheng Han (China)

Paul Pui-Hay But (Hong Kong)

Joseph Tak-Fai Lau (Hong Kong)

Bao-Cang Cai (China)

Chun-Guang Li (Australia)

Kelvin K.-C. Chan (Hong Kong)

Liang Liu (Hong Kong)

Timothy M. Chan (USA)

David Story (Australia)

Pui-Kwong Chan (USA)

Frank Thien (Australia)

Wai-Yee Chan (USA)

Ling-Ling Wang (China)

Il-Moo Chang (Korea)

Kenji Watanabe (Japan)

Yung-Hsien Chang (Taiwan)

Kin-Ping Wong (USA)

Chun-Tao Che (Hong Kong)

Peishan Xie (China)

Chieh-Fu Chen (Taiwan)

Ping Xu (China)

Yung-Chi Cheng (USA)

Bing Zhang (China)

Moses Sing-Sum Chow (Hong Kong)

Zhong-Zhen Zhao (Hong Kong)

Kwok-Pui Fung (Hong Kong) 
This page intentionally left blank 


\section{Contents}

Contributors $\quad$ xiii

Preface to Series $\quad$ xvii

Preface to Volume $4 \quad$ xix

Chapter 1 Healthy Aging: Western and Oriental Means 1 of Accomplishment

Ping-Chung Leung

1.1 Introduction

1.2 Aging-Related Diseases 4

1.3 Healthy Aging 5

1.4 Aging in the Oriental Context 9

1.5 The Concept of Herbal Intake to Support Aging 11

1.6 Use of Chinese Herbs for Prevention of 15 Aging-Related Disease

1.7 Function of Herbs Used for Longevity and 17 Aging Diseases

1.8 Physical Activities $\quad 18$

1.9 Living in Harmony 21

1.10 The Real Situation That We Are Facing 22 References 23

Chapter 2 Study on Thoughts of "Treating Disease Before 25 Its Onset" from Famous CM Doctors

Chun-Bo Xu and Xiang-Yong Zhang

2.1 The Origins of the Thoughts of "Treating Disease Before Its Onset" 
2.2 The Thought of "Treating Disease Before Its Onset" from Famous CM Doctors

References

Chapter 3 Theoretical Study of "Preventive Treatment of Disease" in Traditional Chinese Medicine Lei Hong

3.1 Introduction 41

3.2 The Significances of "Preventive Treatment of Disease" in Health Preservation Science - Self-Regulation During Disease-Free

3.3 The Significance of Preventive Treatment of Disease in Etiology Pathogenesis - Prevention on Pathogenic Factor

3.4 The Significance of Preventive Treatment of Disease 48 in Sub-Healthy

3.5 The Significance of Preventive Treatment of Disease in Sub-Clinical

3.6 The Significance of "Preventive Treatment of Disease" in Clinical Prevention Science

3.7 The Significance of Preventive Treatment of Disease in Clinical Recuperation Science References

Chapter 4 Botanical Supplements for Aging

Harry H.S. Fong and Gail Mahady

4.1 Introduction 56

4.2 Boswellia Resin 58

4.3 Echinacea

4.4 Ginkgo

4.5 Ginseng

4.6 Hawthorn

4.7 Huperzia

4.8 Pygeum

4.9 Saw Palmetto $\quad 83$

4.10 Conclusions $\quad 86$

References 
Chapter 5 Chinese Functional Foods for Aging Individual Choices

\section{Zhi-Xiu Lin}

5.1 Introduction

5.2 Aging from the Perspective of Chinese Medicine 95

5.3 Chinese Medicines Used as Functional Foods 96

5.4 Principles of Using Chinese Functional Foods 98 for Health Promotion

5.5 Chinese Functional Foods for Aging

5.6 Examples of Using Chinese Functional Foods for Aging — Individual Choices

5.7 Discussion

References

Chapter 6 Herbal Formulation for Anti-Aging Song-Ming Liang

6.1 The Theoretical Basis of Anti-Aging and Health Maintenance Drug in Traditional Chinese Medicine

6.2 TCM Drugs and Immunologic Function of the Body

6.3 Important Classical Health Preserving Anti-Aging Formulae

6.4 Formula for Supplementing Yin and Delay of Aging

6.5 Formulas for Supplementing Yang and Anti-Aging

6.6 Health Maintenance in Traditional Chinese Medicine References

Chapter 7 Chinese Herbal Medicine: Perspectives on Age-Related Neurodegenerative Diseases

Khaled Radad, Rudolf Moldzio, Lin-Lin Liu and Wolf-Dieter Rausch

7.1 Introduction

7.2 Role of Chinese Herbal Medicine in Age-Related 
7.3 Other Chinese Herbs

7.4 Concluding Remarks

References

Chapter 8 Insomnia and Aging

Yun-Kwok Wing and Siu-Ping Lam

8.1 Introduction

8.2 Sleep Physiology with Advancing Age

8.3 Insomnia in the Elderly: Clinical Epidemiology

8.4 Insomnia: Comorbidities, Differential Diagnosis and Consequences

8.5 Etiology of Insomnia

8.6 Management of Insomnia in Elderly

8.7 Conclusion

References

Chapter 9 Study on the Mechanisms of Treating Stable Chronic Obstructive Pulmonary Disease with Bufeiyishen Granule

Jian-Sheng Li, Su-Yun Li, Min-Hang Wang and Xue-Qing $Y u$

9.1 Introduction

9.2 Part 1: Study on the Mechanisms of Treating Stable

Chronic Obstructive Pulmonary Disease with Bufeiyishen Granule

9.3 Part 2: The Influence of Bufeiyishen Grain on Bronchial Lung Tissue Metalloprotease in Rats COPD Model

9.4 Discussion

9.5 Conclusions

References

Chapter 10 Dental Disease in the Elderly — From an Integrated Medical Perspective

Sim-Kim Cheng 
10.3 TCM View on Dental Diseases 213

10.4 Oral Diseases as Manifestations of Systemic Disorders 214

10.5 Oral Diseases of Unknown Etiology 216

10.6 Saliva as an Interface for Oral Homeostasis 217

10.7 Effect of Organ Systems on Saliva 218

10.8 Conclusion 220

References $\quad 220$

Chapter 11 Natural Healing in Chinese Medicine: 221

Qi Gong and Tai Chi

Ping-Chung Leung

11.1 Introduction

11.2 Philosophical Background 222

11.3 Concepts of Natural Healing 223

11.4 Practice of Natural Healing 224

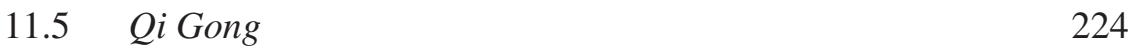

$\begin{array}{lll}11.6 & \text { Tai Chi } & 226\end{array}$

11.7 Clinical Research on Qi Gong and Tai Chi 228

11.8 Conclusions 236

References $\quad 242$

Chapter 12 The Status of Yoga Research in India 245

Shirley Telles and Naveen K. Visweswaraiah

12.1 Early Studies Investigating Special Powers in 245 Accomplished Practitioners

12.2 A Novel Approach: Studies on Less Experienced and 246 Naïve-to-Yoga Persons

12.3 Therapeutic Applications of Yoga 247

12.4 Applications of Yoga in Physiological and Premorbid 251 Conditions

Acknowledgments 254

References $\quad 254$

Index 
This page intentionally left blank 


\section{Contributors}

\section{Sim-Kim Cheng}

Chung Hwa Medical Institution

Singapore College of Traditional Chinese Medicine

Singapore 319522

\section{Harry H.S. Fong}

Department of Medicinal Chemistry and Pharmacognosy

University of Illinois at Chicago

Chicago, IL 60612, USA

\section{Lei Hong}

School of Traditional Chinese Medicine

Chongqing Medical University

Chongqing, China

\section{Siu-Ping Lam}

Department of Psychiatry

Shatin Hospital

Shatin, New Territories, Hong Kong

\section{Ping-Chung Leung}

The Institute of Chinese Medicine

The Chinese University of Hong Kong

Shatin, New Territories, Hong Kong

\section{Jian-Sheng Li}

Gerontology Institute

Henan Traditional Chinese Medical College

Zhengzhou, Henan 450008, China 


\section{Su-Yun Li}

Institute of Respiratory Disease

First Affiliated Hospital of Henan Traditional Chinese Medical College Zhengzhou, Henan 450008, China

\section{Song-Ming Liang}

School of Chinese Medicine

The Chinese University of Hong Kong

Shatin, New Territories, Hong Kong

\section{Zhi-Xiu Lin}

School of Chinese Medicine

The Chinese University of Hong Kong

Shatin, New Territories, Hong Kong

\section{Lin-Lin Liu}

Key Lab for Supramolecular Structure and Materials

Jilin University, Changchun 130012, China

\section{Gail Mahady}

Department of Pharmacy Practice

PAHO/WHO Collaborating Center for Traditional Medicine

College of Pharmacy, University of Illinois at Chicago

Chicago, IL 60612, USA

\section{Rudolf Moldzio}

Institute for Medical Chemistry

Veterinary Medical University

Vienna 1210, Austria

\section{Khaled Radad}

Department of Pathology, Faculty of Veterinary Medicine Assiut University, Assiut 71526, Egypt

\section{Wolf-Dieter Rausch}

Institute for Medical Chemistry

Department of Natural Sciences

University of Veterinary Medicine Vienna

Vienna 1210, Austria 


\section{Shirley Telles}

Swami Vivekananda Yoga Research Foundation

Bangalore, India

Naveen K. Visweswaraiah

Foundation for Assessment and Integration of Traditional Health Systems [FAITHS]

Bengaluru, India

\section{Ming-Hang Wang}

Gerontology Institute

Henan Traditional Chinese Medical College

Zhengzhou, Henan 450008, China

\section{Yun-Kwok Wing}

Department of Psychiatry

The Chinese University of Hong Kong

Shatin, New Territories, Hong Kong

\section{Chun-Bo Xu}

World Federation of Chinese Medicine Societies Xianlin University City

Nanjing, Jiangsu Province, 210029 China

\section{Xue-Qing Yu}

Gerontology Institute

Henan Traditional Chinese Medical College

Zhengzhou, Henan 450008, China

\section{Xiang-Yong Zhang}

World Federation of Chinese Medicine Societies Xianlin University City

Nanjing, Jiangsu Province, 210029 China 
This page intentionally left blank 


\section{Preface to Series}

\section{Does Traditional Chinese Medicine Work?}

History should be acknowledged and respected. Despite this, the historical value of Chinese medicine in China and some parts of Asia should not be used as the only important evidence of efficacy.

While clinical science has followed closely the principles of deductive research in science and developed its methodology of wide acceptance, there is a natural demand from both users and service providers that the same methodology be applied to the traditional art of healing. There should be only one scale for the measurement of efficacy. Thus, evidencebased medicine, which apparently is the only acceptable form of treatment, would also claim its sovereignty in Chinese medicine.

In spite of influential proponents and diligent practitioners, efforts relating to the application of evidence-based medicine methodology to Chinese medicine research have been slow and unimpressive. This should not come as a surprise. Evidence-based medicine requires the knowledge of the exact chemistry of the drug used, the exact physical or chemical activities involved and above all, the biological responses in the recipient. All these are not known. Working back from the black box of old historical records of efficacy requires huge resources and time, if at all possible. Insistence on this approach would result in either unending frustrations or utter desperation.

Parallel with the modern attempts, respectable Chinese medicine practitioners have unendingly and relentlessly cried out their objection to the evidence-based approach. They insisted that all the evidences were already there from the Classical Records. Forcing the classical applications through a rigid modern framework of scrutiny is artificially coating Chinese medicine with a scientific clothing that does not fit. 
Thus, the modern proponents are facing an impasse when they rely totally on modern scientific concepts. The traditional converts are persisting to push their pilgrims of defense. Where do we stand so as to achieve the best results of harmonisation?

There must be a compromise somewhere. Classic evidences can be transformed into a universal language to be fairly evaluated and to be decided whether suitable for further research, using the deductive methodology or an innovative one after intelligent modifications.

There is a need for a platform on which a direction can be developed in the attempt to modernise the traditional art and science of healing, while remaining free and objective to utilise the decaying wisdom without prejudice.

With the growing demand for complementary/alternative medicine from the global public and a parallel interest from the service providers, there is an urgent need for the provision of valuable information in this area.

The Annals of Chinese Medicine is a timely serial publication responding to this need. It will be providing authoritative and current information about Chinese medicine in the areas of clinical trials, biological activities of herbs, education, research and quality control requirements. Contributors are invited to send in their reports and reviews to ensure quality and value. Clinicians and scientists who are willing to submit their valuable observations, resulting from their painstaking researches are welcome to send in their manuscripts. The Annals of Chinese Medicine has the objective of providing a lasting platform for all who concentrate their efforts on the modernization of Chinese medicine.

\section{Professor Ping-Chung Leung}

Institute of Chinese Medicine The Chinese University of Hong Kong 


\section{Preface to Volume 4}

The fourth volume of the Annals of Traditional Chinese Medicine is dedicated to Aging. With affluency the projected lifespan of people in every developed or developing country increases. Longevity is considered a blessing worldwide, more emphatically among the Oriental communities. Living a long and healthy life is on everyone's wish list, whereas being an elderly with chronic diseases and multiple degenerative areas is our greatest fear. From the socio-economic perspective, caring for an evergrowing elderly population is in itself a highly challenging responsibility on both community and government levels. Caring for the same number of unhealthy individuals will be an almost impossible task.

Ever since the elderly boom has become real, health providers and bioscientists have been directing a lot of resources to the maintenance of health for the elderlies. Aging and degeneration are coupled facts. Deterioration of physical ability leads to frailty. If there are ways to slow down degeneration and practices to maintain physical ability, general and widespread promotion should start immediately.

Oriental societies have a long history of promoting health by preventing disease, which are not short of special terms and connotations. Thus for traditional Chinese medicine (TCM), "treating diseases before its onset" does not refer to what we understand by the Public Health Standard of disease prevention, but points to a more personalized pursuit of good health before the individual is disease-stricken. Under this category of medical practice, living and eating habits, lifestyles and special supplements, are all included. In fact TCM practitioners consider food to be a form of medicine. The Indians are equally conscious about lifestyle and diet. 
The Oriental concept of health is gradually being recognized by the affluent societies in the US and Europe to different degrees as are apparent from the rising popularities of Oriental health supplements and exercises. However, with the highly developed advances in science, the main approach to geriatrics is still geared towards scientific discoveries and technological interventions which are linked with new enterprises.

Different commercial health promotion groups and societies have emerged in the past ten to 15 years, offering high-level investigations for people who are elderly or have reached a certain age, to "rule-out" causes of ailment. Logically speaking, ailments may not be felt (symptomatic), but probably will further deteriorate into illnesses. These health enterprises label the ailments as mild or subclinical deficiencies, or excesses. The manifestations can be related to exocrine glands, hence causing gastrointestinal ailments; or working through endocrine systems, thus producing minor hormonal disturbances. Likewise food allergy is equated with toxicity, or subclinical toxicities which are assumed to be affecting the whole individual, not only confined to the digestive system. Sophisticated laboratory tests are required because the clinical laboratories are capable of detecting only gross deficiencies, excesses and toxicities. The highly specialized tests are aimed only at amino and organic acids, which are carbohydrate metabolism markers, methylation pathway markers, antioxidant markers, intestinal dysbiosis markers, fatty acid metabolism markers, etc. These highly specific chemicals are detected from the individual's blood or urine samples. These groups of clinicians and bio-scientists have extrapolated their laboratory knowledge to the clinic; so when a certain metabolic pathway goes off track, they come to expect some marker change. However, there are those of us who realize that our day-to-day physiology is actually a dynamic process that constantly changes and yet is maintained in a broad level of balance or harmony. They have doubts on whether a unique, queer, minor diversion from the normal pathway is serious enough to warrant treatment. Thus, the new health enterprises targeting towards minor metabolic changes can be charged with the engagement in medicalization.

It is therefore the intention of this book to devote the contents to the Oriental way of maintaining elderly health: from the general concepts, to eating habits; from botanical supplements, herbal medicine, to natural healing in China and India. Some specific considerations are given to some 
areas like neurodegeneration, sleep disorders, pulmonary obstruction and dental problems. Perhaps the best way to prepare for a healthy longevity is to be able to formulate a do-it-yourself program.

As always the Editors are most grateful to the generous contributions of the authors.

\author{
Professor Ping-Chung Leung \\ Institute of Chinese Medicine \\ The Chinese University of Hong Kong
}

